many interesting questions in my mind, which I can at present only mention, notwithstanding their probable deep importance.

r. What is the interpretation of this apparent increased functional activity of the kidneys in functional albuminuria?

2. Does the cause lie in the kidney itself, or is it the expression of some change of function in a remote organ; or is it the result of changes in the normal metabolism of the blood?

3. What are the relations of this increased specific gravity of the urine to functional albuminuria?

We may perhaps assume that the cause of the increased specific gravity of the urine, and perhaps of the albuminuria itself, are external to the kidneys; since, as has been shown, disease of those organs invariably results in reducing the specific gravity of the urine. May not regressive changes in the economy furnish not only an excess of solids to be eliminated by the kidneys, but also in the splitting up of organic compounds result in the formation of a proteid so altered as to permit its escape through the renal filter, while yet not sufficiently altered to change its properties of responding to our ordinary tests for albumin?

There are a number of circumstances favoring such a view. Our lack of knowledge of the chemistry of proteids or albumins; together with the wellknown facts that they are capable of very great changes in the line just suggested, favor this view. It is well known, for instance, that when albumin has taken certain regressive steps in organic metamorphosis, it is at once eliminated by the kidneys, as is observed in peptonuria and propeptonuria. Now, may not less marked changes in proteids-say an intermediate step between albumin and peptoneresult in the formation of a proteid which answers to all the properties suggested? It seems to me that such is quite possible, if indeed not probable.

In a very valuable article on "Functional Albuminuria and its Relation to Hæmoglobinuria" Dr. Ralfe, ${ }^{4}$ of London, has made some suggestions on the close relationship between accelerated metabolism of the blood- "increased hæmolyses"- and functional albuminuria.

As is now known, the red corpuscles of the blood are broken down in the liver. The hæmoglobin is converted into pigment and urea in health. The albuminous element of the blood corpuscles is converted in the liver into urea ordinarily; but if the destruction be abnormally great Dr. Ralfe believes that " a large portion of the albumin passes off by the kidneys." Increased hæmolysis would undoubtedly account for the rise in the specific gravity of the urine in these cases, for with increased hæmolysis we have increase of urea, and the latter constitutes about onehalf of the solid urine.

Now to the various laws formulated in this paper I am aware of but one exception, and that occurs in cases of chronic Bright's disease combined with diabetes mellitus. The occurrence of such a combination is, I believe, much more common than is gener-

\footnotetext{
${ }^{4}$ British Me 1. Jour. Nov. $27,1886$.
}

ally supposed. It is true that we are accustomed to find in chronic diabetes small quantities of albumin in the urine, and this is usually attributed to the irritation in the kidneys induced by the excretion of sugar. The number of cases of diabetes, however, which have come under my observation accompanied by slight albuminuria and which have subsequently proved to be conjoined with chronic Bright's disease have induced me to adopt the following rule: In every case of diabetes accompanied by slight albuminuria, if the patient's age be over forty years, search carefully for evidences of contracting kidney. The investigation will present points of unusual difficulty, since the increased volume of urine greatly lessens the chances of discovering renal casts-which are indeed always sparse and difficult to find in this disease. The vascular tension is markedly increased in diabetics, which takes away another differential diagnostic prop in such cases; and lastly, as already indi. cated, the specific gravity of the urine is much above normal. It will therefore be observed that the investigation must be most thorough in these cases.

The normal urinary solids, as we have seen, are always reduced in chronic Bright's disease, and therefore ordinarily the specific gravity of the urine falls below normal. The excretion of sugar, however, often to the extent of 25 to 40 grains in each ounce of urine in diabetics, more than counterbalances the deficiency in solids induced by interstitial nephritis; and therefore the specific gravity of the urine must necessarily rise considerable above the normal standard when the two diseases occur in conjunction. It will not answer, therefore, always to attribute the slight albuminuria in diabetes to simple renal irritation, or consequences the most grave and disastrous, which might otherwise be averted, will sometimes overtake these cases.

r63 State street.

\section{SUDDEN DEATH IN LABOR AND CHILDBED.}

Read before the Section on Obstetrics and Gynecology, at the Thirty-eighth Annual Meeting of the American Medical Association, Fune, 1887 .

BY FAYETTE DUNLAP, M.D., OF DANVILLE, $\mathrm{KY}$.

On January I 4, I887, I was summoned to attend a lady, aged 24 years, in her first confinement. I had been engaged some months before, and by reference to my visiting book I find that she was expected to be confined on January 27 . The note summoning me stated that there had been a slight hæmorrhage from the vagina for several hours, and that a premature delivery was imminent. An examination proved that labor had begun, and the os was dilated to an extent to admit the index finger. There were no distinct contractions, but the os was slowly dilating, and very satisfactorily. The hæmorrhage continued constantly but scantily. I ordered a warm vaginal douche, left the house and returned after four hours. There had been some progress made, the parts were splendidly lubricated, the pains coming on periodically and increasing in force-in a few words, the labor was progressing naturally. 
$I$ retired in an adjoining room at Io P.M. with instructions to be called at 2 A.M. At this hour my patient was sleepless, suffering acutely, and the expulsive efforts very feeble, whereupon I gave hypodermatically $1 / 6$ gr. morphia and $\frac{1}{150}$ gr. atropia. She slept quietly for a short time, and by 7 A.M. the contractions began in earnest. From the beginning of labor the suffering was acute, yet the woman showed no evidence of exhaustion and was very hopeful of a satisfactory issue out of her confinement. I gave $30 \mathrm{gr}$. of chloral per rectum, as the rim of the os was very sharp and unyielding and the parts becoming dry. I could not perceive that it had the least effect locally or constitutionally, and repeated the hot water douche at I I A.M.

At I P.M. the delivery was completed and the woman seemingly in unusually good condition, considering the number of hours she had been the victim of acute suffering. At no time was there the slightest despondency or evidence that her nervous system was influenced by it. The perineum relaxed well, and there was no more than the customary amount of laceration for first labors. The placenta was expelled without traction or great amount of compression, and it is absolutely certain that there was no bleeding of consequence. I gave my patieni my undivided attention for twenty minutes or more, and save a pulse-beat of about roo and very great prostration there were no unfavorable symptoms. She had not moved from her position and was breathing tranquilly.

I was preparing to leave the room when she asked me to return, as she felt as if about to faint. I instantly thrust my hand into the vagina, expecting hæmorrhage, but found the womb firmly contracted and plainly to be outlined above the pubes. Dyspnoea, nausea and a turbulent tossing about in bed began. The second sound of the heart was lost, and the organ in a flutter and not to be counted. I grasped the shoulders and lowered the head almost to the floor, and in a few seconds consciousness returned, and with it an improved tone of the heartbeat. I replaced her quietly in bed, and gave $\mathbf{I}$ ounce of brandy and had the body warmed. At this juncture I despatched a messenger for a consuitant, and in a few minutes Dr. McMurtry arrived. As the heart's action was feeble and the countenance livid, I gave every few minutes brandy hypodermatically for an hour.

The tone of the heart's beat would improve and remain strong a few moments at a time, and would then gradually fade away and syncope would again follow. The dyspnœa continued, the cyanosis was alarming, and the surface was shrunken and freely perspiring. Precisely four hours after the first syncopic seizure the woman suddenly lifted herself in bed, uttered a faint scream and fell back dead. Her mind was clear up to the moment of death.

In prefacing my remarks upon this case it may be well to state that is my custom to make an analysis of the urine of my pregnant patients, usually about one month before confinement. As there was considerable œdema of the ankles, I examined the urine three times microscopically and chemically. She was a strong, robust, hopeful person, and had a remarkably fine family history.

This sad tragedy, the first one that I had witnessed, made a deep impression upon me, and I repaired at once to all the obstetric literature at my command for some explanation of this unexpected occurrence. The very meagre amount to be found in even the most popular and practical works was a matter of surprise. Leishman gives about one-fourth of a page, and simply says that very rarely a patient suddenly dies in childbed from some unknown cause, such as shock, heart-failure or exhaustion. Playfair gives something more; Cazeaux and Ramsbotham and Parvin nothing at all. It remains for Lusk, who had an experience similar to my own just prior to giving his work to the press, to give especial attention to this subject. A few weeks ago I reported this case to the Medical Society of my village, and nearly every practitioner had had one or more lying-in women suddenly die with the assemblage of symptoms above related. A calamity of this kind, if taking place only once on an average in the professional life of every general practitioner, deserves more attention than has been given to it.

This, and a like experience to be related hereafter, brought the matter prominently forward in $\mathrm{my}$ studies. In prefacing his remarks upon the class of cases to which this history belongs Winckel says: "He who has once seen a puerperal woman happy and joyous in the expectation of soon leaving her couch, then directly afterwards has found her a corpse, will be able to comprehend the truth of Hervieux's words: 'In the twinkling of an eye all the calculations of prudence are set at naught; the most untiring vigilance, the best established rules of hygiene, all the varied resources and ingenious combinations of therapeutics, are shattered against an invisible rock;" "then adds with a tone of despondency: "The only thing which remains for the physician after such a depressing occurrence is to seek out the cause of the sudden death."

In this search it is my purpose to pass over consideration of rupture of the uterus, apoplexy, the snapping asunder of an aneurism and post-partum hæmorrhage, and following the example of Lusk, confine myself to entrance of air into the lungs, shock, exhaustion and embolism.

The surgeons have taught us that the entrance of air into the venous circulation is fraught with danger. This accident is most likely to occur in opening the veins of the neck, the act of inspiration encouraging the entrance of a volume of air into the open venous mouths. The sudden emptying of the womb produces a like result, and it is plainly to be understood how air can find its way into the open veins of the placental site. This is particularly true in cases in which the labor has been unduly prolonged and the uterus left in a relaxed condition. It has frequently happened that sudden death has occurred before delivery was accomplished, this being due to the fact that a partial separation of the placenta has left the venous sinuses standing widely open. The plan of inducing premature delivery recommended by Kiwisch, then, is particularly perilous. 
I can recall the particular features of a case in my forced the head to the os externum, and caused the practice which seems to illustrate the dangers from placental separation. The next contraction caused this source: I placed my patient in the Sims' posi the uterine air to escape by the only channel that tion, introduced his speculum in order to remove a was possible, viz.: by the open mouths of the plafour months' foetus that was firmly held by the rim of cental veins."

the os uteri. I grasped the mass with the placental forceps and easily dislodged it. After removing the secundines with the forceps I introduced the nozzle of a Davidson's syringe and forced in a stream of water to clear the womb of clots and débris generally. Pure, very warm water only was used. The os seemed sufficiently relaxed to allow an unobstructed return of the water thrown in. While this was in progress the woman began to breathe heavily, spoke in a whisper, and became cyanotic. She felt as if a heavy weight was bearing her down. The effort to breathe was violent, and she breathed more rapidly than I thought it possible for an adult to do. The heart was beating tumultuously, but strong enough. I used brandy freely hypodermatically, and even ventured upon giving 20 drops of liq. ammon. in one syringeful. After lying a few minutes in a relaxed and semi-unconscious state she slowly revived and made a prompt recovery. Sir James Paget tells us that it is possible for a considerable amount of air to enter the circulation, and if it is properly disposed through the lungs it will be speedily absorbed and do no damage. I have always believed that the sudden and firm contraction of the uterus caught above the internal os a volume of air that had been forced in by the syringe, and it found its readiest exit by way of the venous sinuses. My father, who was holding the speculum, remarked that the symptoms presented an exact counterpart to those in a man dying on the operating table from the entrance of air while undergoing an operation for exsection of a portion of rib.

Lusk quotes an interesting case reported by Kezmansky, of Budapest. The patient was $3^{6}$ years of age, and in labor for the fourth time. An excessive amount of fluid was recognized. The patient entered the hospital at Io A.M. At 3 P.M. she lay upon her left side, and the bag of waters protruding from the vulva. Spontaneous rupture occurred and a gallon of water escaped. Five minutes later the patient suddenly threw back her head, gasped a few times for air, the face became livid, and respiration was arrested. In scarcely two minutes Dr. Liebman was at her side, but the pulse was extinct. The forceps were applied and an asphyxiated child was delivered. The heart was feebly beating, and the woman died before delivery was completed. At the post-mortem examination, twenty hours after, pale reddish-brown blood, mingled with bubbles of air, was found in the uterine veins, vena cava, lungs, and especially in the pulmonary artery. In the vena cava, columns of blood and air alternated. Kesmansky makes the following statement in his report: "The patient lay upon her left side with her knees drawn up in nearly the Sims' position, with the vulva elevated above the concavity of the abdomen. As the membranes rup. tured there was a sudden diminution of the intraabdominal pressure, so that the air entered through the open vulva between the collapsed membranes and the uterus. The ensuing, retraction of the uterus

In the St. George's Hospital Reports, vol. vi, Cordment reports two cases. Frcm the position of the patients at the time of delivery it was an easy matter for air to gain admission. Post-mortem examination demonstrated that the larger veins in both the abdominal and thoracic viscera had bubbles of air in them, and in the right side of the heart. The brain was anæmic and contained frothy serum. In addition to these symptoms was the extreme pallor of the surface, feeble pulse, jerky and rapid respiration and speedy collapse.

It is thus demonstrated that Kiwisch's plan of inducing premature labor by the forced stream of water; that to admit a sudden volume of air into the womb while the sinuses are open; to allow the woman to be delivered in the standing position; to deliver her in the knee-chest position, as suggested by Ludwig under certain contingencies, are dangerous in the extreme. In my case I can never be certain that air in the veins was the cause of the alarming condition, but I shall always believe so. The sudden onset of the symptoms, their sudden disappearance and the favorable position of the patient, are the only evidences I have to offer to sustain my belief.

I now turn to a consideration of shock or exhaustion as a cause of sudden death in childbed. The phenomena of surgical shock are typically illustrated in the case reported at the beginning of this paper, and are almost exactly reproduced in a case within the last month. I was hastily called in consultation by Dr. McMurtry, who had an hour previously delivered his patient of her seventh child. She was 42 years of age, very robust and an illustration of perfect health. By overtaxing her strength in household. duties labor was induced two or three hours in advance of maturity. Labor came on rather precipitately, but aside from this, it was completed without an untoward symptom. She informed her attendant that in her last confinement the hæmorrhage was alarmingly profuse. Immediately after the expulsion of the foetus, and before the placenta had been detached, Dr. McMurtry noticed the pallor of countenance and the complete relaxation of the whole muscular system. The heart was beating rapidly; could not be counted, and the second sound was obliterated. The patient lay quietly, breathed easily, and presented a typical picture of surgical shock. A noticeable feature was the very marked improvement at times of the tone of the circulation, and its sudden recession within a few seconds' time. There were no convulsions, no loss of blood, and the only complaint was that of præcordial distress. Dyspnoea came on gradually, and in exactly five hours from delivery she quietly passed away. This was an almost exact reproduction of $m y$ first experience, and differed only in this, that in the first patient restlessness and great distress were prominent, and in the second they were not present at all.

In the absence of any demonstrable evidence of 
the presence of air in the veins or of embolism, we must attribute death to that mysterious complexus of symptoms we include in the general term of shock. Pathological investigation refers the phenomena of shock to a reflex paralysis of the vaso-motor or splanchnic nerves, whereby the mass of the blood recedes from the surface and collects in the thoracic or abdominal viscera. Through this same influence the heart is enfeebled, and soon overworked by its ineffectual efforts at ridding itself of its constantly increasing burden, and finally yields to the pressure and ceases to contract. The condition is favorable to coagulation, and doubtless this often occurs.

In addition to the pains and exhaustion of childbirth, mental emotions have no small part in the production of a state of the nervous system favorable to shock. The patient had been for a year brooding over a domestic tragedy, and made frequent visits to the State Prison to see her son, who was undergoing a sentence for murder. She was greatly humiliated, lost sleep, and was ready at all times to give way to the most wretched despondency. How great a part this circumstance had in bringing about the calamity we are unable to say. Lusk tells us that the homesick prisoner of war and the conquered soldier who is left on the field of battle yield with readiness to shock and perish from the most trivial wounds. We cannot ignore these facts, and must give prominent place in our classification to that at present unexplainable something we call shock.

Unless the case reported at the beginning as an introduction was one of embolism, I have never encountered the accident in my midwifery practice. As an autopsy was denied, the diagnosis is made solely upon the symptoms above detailed, and moreover, I cannot find a single report of an embolus having been discovered in an autopsy of a patient dying during or immediately after delivery.

It has been assumed for a number of years that the blood is in a favorable state for coagulation during pregnancy. This theory has been strongly maintained by Barker, Playfair, Meigs and others, and in clinical experience many striking facts have arisen to support this belief. Dr. Mary Putnam Jacobi reported a case similar to my first one to the New York Pathological Society, and exhibited the heart containing the clots, which she maintained were post-mortem.

In all the cases reported the feeble circulation was always the first symptom to cause alarm, and, if coagulation takes place it is the result of this enfeebled circulation, and not its cause. The fact remains that numbers of puerperæ die and with symptoms as detailed above in these three cases. While there is great similarity in them, yet they are widely apart in many important particulars.

Now, it is not sound pathology to ignore these facts and to banish as unworthy of consideration a well established pathological condition, simply because in the days of ignorance the terms exhaustion and shock were indiscriminately employed to explain a multitude of cases which, with our present enlightenment, we know are due to the entrance of air into the circulation, and to pulmonary embolism. As the nervous organization of woman loses in powers of resistance as the penalty of a higher civilization and of artificial refinement, it becomes imperatively necessary for the physician to guard her from the dangers of excessive and too prolonged suffering. I have always urged the principle and have carried it out in practice, that the exquisite torture to which many women are subjected is unnecessary. The impression made upon a sensitive organization by prolonged and constantly increasing pain can and does result in damage to the central nervous system. In a measure, then, we can diminish the liability to danger from shock and exhaustion by timely and judicious interference, to danger from entrance of air into the circulation by the position of the patient, the means employed in assisting uterine dilatation, but in the prevention of clot formation, in the light of our present pathological knowledge, we are powerless, and in the presence of either of these conditions we must stand helplessly by and say with Winckel, that the only thing remaining for the physician to do is to seek out the cause of death.

\section{DISCUSSION}

DR. I. N. Rosenthal, of Fort Wayne, Ind. : Sudden deaths after parturition are fortunately very seldom, but they are nevertheless very terrible, and I hope to be excused if I report a case in full. A multipara was taken sick and confined about 8 P.M., and about 3 A.M. I was called by the attending physician. The lady was in a perfect frenzy of anxiety because the attending physician had stated to the parties that there was something wrong. I found a second fotus in shoulder presentation, with no worse symptoms than would naturally be expected in a case of malposition or where labor was not normal. I turned the child without trouble, and after delivery the lady seemed to be perfectly happy and satisfied, and expressed feelings of gratitude and comfort. Within a half hour the attending physician and myself noticed the pulse becoming less, the lady felt as if she was fainting, but there were no other symptoms; the uterus was perfectly contracted, and no sign of hæmorrhage. The pulse continued to become weaker, and in less than half an hour the woman collapsed and death took place. What was the cause of death if it was not thrombosis or embolism? It certainly was not shock, there was no sign of sufficient shock for at least half an hour after delivery. DR. W. W. GRanT, of Davenport, Iowa: There is one point of much interest in connection with this case which I do not think ought to be passed by unnoticed, and that is, whether we are as helpless as the author of the paper would indicate. It seems to me that in these cases of accidental hæmorrhage due to separation of the placenta, as distinguished from placenta prævia, it is just one of those slight hæmorrhages that come on previous to delivery. The hæmorrhage in this case was probably the cause of premature death, and is a very interesting point. These cases are not so uncommon, and it is a question whether we cannot do something to arrest that condition. I have recently had such a case, and have treated other cases, and I think something can be done to avert the embolism, whether it is from 
air or blood, and that is the administration of ergot, even before labor in this case; I have done it systematically, have given ergot not in large enough doses to produce labor, but enough to produce a gentle, firm contraction of the uterus. I gave it recently for three weeks, 25 drops Squibbs' extract of ergot. That contraction is worth something even after delivery.

Dr. W. H. WAthen, of Louisville, Ky.: I was certainly very much interested in this excellent paper read by Dr. Dunlap. I was specially interested because I have had a misfortune somewhat similar recently, in which a lady was sent to me from a distance who had become illegitimately pregnant. She came of a very good family, was very much depressed, and in fact had no desire to live. She passed through her delivery, and in the second stage of labor the uterus contracted down and the placenta was removed without difficulty, and it contracted again. In about five minutes hæmorrhage began and the uterus relaxed. I introduced my hand very quickly and grasped the uterine cavity and the hæmorrhage ceased. The woman lost probably eight or ten ounces of blood. A hypodermic injection of ergot was given immediately. We had no warm water, nothing but a solution of chloride of iron, one part iron to ten warm water. The hæmorrhage immediately ceased, the uterus contracted down firmly, and remained contracted. The woman did not bleed any more, her pulse was perfectly good, her respiration normal, no disturbance of any sort whatever. In probably half an hour after this I felt the pulse and it was getting rapid; it had been 70 to 80 , then 90 , then it went up to 100 ; finally she was gasping for air, could not breathe, the pulse I Io, and it finally disappeared altogether. I gave hypodermic injections of whiskey, but she gradually grew worse, rallied again with external heat, grew worse, and in an hour died, remaining conscious until probably ten minutes before death. This is a case in which it is difficult to understand the cause of death. It certainly was not from shock; there was no apparent shcck during labor or immediately after, and no apparent shock after hæmorrhage had ceased. I could not account for it by air in the blood-vessels, because if air had got in it would have been when the injection was used, and symptoms would have been manifested immediately. I accounted for it by pulmonary clot, embolism, where probably the clot from the uterus was by some means taken up through the vena cava to the heart and then to the lungs. If it was not a pulmonary embolism I am unable to account for it.

\section{WOUND DRESSING : SOME NOTIONS ACCEPTED AND SOME UNDER DISCUSSION.}

Read in the Section on Surgery and Anatomy, at the ThirtyEighth Annual Meeting of the Am Medical. Association. BY GEORGE E. STUBBS, A.M., M.D.,

PROFESSOR OF SURGICAL, PATHOLOGY IN THE MEDICO-CHIRURGICAL COZ LEGE, AND SURGEON TO THE CHARITY AND THE MEDICO-CHIRURGICAL HOSPITALS, OF PHILADELPHIA.

In presenting a few thoughts upon the subject of wound dressing, I do not feel that I need to apologize on account of the triteness of the subject, since every surgeon of experience has ideas peculiar to himself on this practical subject, which should be cast in the common fund of information, to add if possible to the heritage of knowledge, which we have jointly received from the honored surgeons of the past. I must, however, disclaim the idea of offering any notions that are very original, though I hope that from an experience of some twenty five years of military and civil surgery that I may present some ideas which may interest for a few moments, if they do not edify.

But even if one cannot always present new and original ideas, as the result of patient, methodical research, it is often of great advantage both to the reporter and the profession to whom he reports, for the physician to take time to put himself upon a point of observation, and take a general view of ground gone over and of prospects ahead. In doing this, I am impressed at once with the rapidity of professional unification. Where less than a generation ago surgeons in each country were a law unto themselves in the matter of surgical tenets, now, so closely do electricity and steam unite the World that the surgeons of every land are our brethren, and if Horsley of England removes a tumor from the brain, and his diagnosis and prognosis are both thereby verified, we clap him on the shoulder, in spirit at least, and hail him as our brother; if Billroth boldly takes the risk and operates for cancer of the pylorus, we fraternally admire his courage and zeal, and resolve to emulate his example as occasion demands; and when Keith presents his latest and best statistics in ovariotomy, we are ready to cry "bravo!"

With this diversion from my subject, let me now remark, firstly, that our ideas upon the subject of wound dressing are constantly changing, I find, on referring to many works of standard excellence in surgery, which grace my library, including the three immense tomes of the "Surgical History of the Rebellion," that many of the terms now constantly in use in our surgical literature, our surgical clinics and the surgical wards of our hospitals, and likewise in every meeting and discussion of well read medical men, are not even mentioned; indeed, such is the rate of progress, especially in the line of bacteriology, that surgical works published but five or ten years ago are now considerably behind the times.

Most of the principles, however, of such works are still as true as ever, so far as experience has yet proven, just as we may still assert, that many of the principles handed down to us from Hippocrates, such as the application of bandages, and, in all severe wounds, rest, a strict regimen, and an easy position for the limb, the rejection of oil and all moist applications except poultices in certain cases.

If I take up a certain standard work on surgery, I find under the general heading of wounds, the indications given thus :

Ist. "The arrest of hæmorrhage," which probably all surgeons now would agree to at once, although Hippocrates believed in letting the blood flow copiously when situated upon the limbs or penetrating the cavities of the body. 